\title{
Temporal and Periodic Variations of Sunspot Counts in Flaring and Non-flaring Active Regions
}

\author{
A. Kilcik ${ }^{1}$ - V. Yurchyshyn',3 . B. Donmez ${ }^{1}$. \\ V.N. Obridko ${ }^{4}$ - A. Ozguc ${ }^{5}$ - J.P. Rozelot ${ }^{6}$
}

(C) Springer $\bullet \bullet \bullet$

Abstract We analyzed temporal and periodic behavior of sunspot counts (SSCs) in flaring ( $\mathrm{C}, \mathrm{M}$, or X class flares), and non-flaring active regions (ARs) for the almost two solar cycles (1996 through 2016). Our main findings are as follows: i) The temporal variation of monthly means of daily total SSCs in flaring and non-flaring ARs are different and these differences are also varying from cycle to cycle; temporal profile of non-flaring ARs are wider than the flaring ones during

\footnotetext{
$\varangle$ A. Kilcik

alikilcik@akdeniz.edu.tr

V. Yurchyshyn

vayur@bbso.njit.edu

B. Donmez

burcindonmez@akdeniz.edu.tr

V.N. Obridko

obridko@mail.ru

A. Ozguc

ozguc@boun.edu.tr

J.P. Rozelot

jp.rozelot@orange.fr

1 Akdeniz University Faculty of Science, Department of Space Science and Technologies, 07058, Antalya, Turkey

2 Big Bear Solar Observatory, Big Bear City, CA 92314, USA

3 Korea Astronomy and Space Science Institute, 776 Daedeok-daero, Yuseong-gu, Daejeon, 305-348, South Korea

4 Pushkov Institute of Terrestrial Magnetism, Ionosphere and Radio Wave Propagation of the Russian Academy of Sciences (IZMIRAN), Troitsk, Moscow, 142190 Russia

$5 \quad$ Kandilli Observatory and Earthquake Research Institute, Bogazici University, 34684 Istanbul, Turkey

6 Université de la Côte d'Azur (OCA-CNRS) and 77, Ch. des basses Moulieres, 06130 Grasse (F)
} 
the solar cycle 23, while they are almost the same during the current cycle 24 . The second peak (second maximum) of flaring ARs are strongly dominate during current cycle 24, while this difference is not such a remarkable during cycle 23. The amplitude of SSCs in the non-flaring ARs are comparable during the first and second peaks (maxima) of the current solar cycle, while the first peak is almost not existent in case of the flaring ARs. ii) Periodic variations observed in SSCs of flaring and non-flaring ARs are quite different in both MTM spectrum and wavelet scalograms and these variations are also different from one cycle to another; the largest detected period in the flaring ARs is 113 days, while there are much higher periodicities (327, 312, and 256 days) in non-flaring ARs. There are no meaningful periodicities in MTM spectrum of flaring ARs exceeding 45 days during solar cycle 24, while a 113 days periodicity detected from flaring ARs of solar cycle 23. For the non-flaring ARs the largest period is 72 days during solar cycle 24 , while the largest period is 327 days during current cycle.

Keywords: Sun: Active Regions, Sunspots, Flares, Periodicity

\section{Introduction}

Our sun is a variable star and it shows different kind of variations such as sunspots, solar flares, prominences, coronal mass ejections etc., as depending on the observed wavelength and depth. These variations can be traced easily by observing such solar activity indicators, as sunspot numbers (SSNs), sunspot areas (SSAs), $10.7 \mathrm{~cm}$ solar radio flux (F10.7), solar flare index (FI), total solar irradiance (TSI), etc. All of these indicators describe solar activity quite well. Due to the longest temporal coverage (about 400 years), sunspots are one of the most important and commonly used indicators. Studies on variations in solar activity are important for understanding the mechanism behind the solar activity and solar cycle, and also for predicting the level of activity (Hathaway, 2009; Petrovay, 2010). The properties of solar cycle are generally described by the Zurich or international sunspot number, $\mathrm{RZ}=\mathrm{k}(10 \mathrm{~g}+\mathrm{f})$, where $\mathrm{k}$ is a correction factor for the observer, $\mathrm{g}$ is the number of identified sunspot groups, and $\mathrm{f}$ is the number of individual sunspots. According to this equation daily sunspot number strongly related to the number of observed group for a day.

In the photosphere the presence of a strong magnetic field is manifested by the appearance of dark sunspots or pores and bright faculae representing concentrated and dispersed magnetic fields, respectively. In general, sunspots are observed as groups on the active regions (ARs) of solar surface and they are classified according to their morphology and evolution. The ARs may be classified in terms of the morphology of the sunspot groups. The most common classification of ARs was introduced by McIntosh (1990). The McIntosh Sunspot Classification Scheme (MSCS) assigns three descriptive codes characterizing the size (A, B, C, D, E, F, H), penumbra (X, R, S, A, H, and K) and compactness (X, $\mathrm{O}, \mathrm{I}$, and $\mathrm{C}$ ) of ARs. The simplest ARs have bipolar magnetic field configurations, but ARs may be built-up by several bipoles emerging in close succession. In this classification A and B classes describe very tiny small SGs and they produce very 
rare flare, while $\mathrm{D}, \mathrm{E}$ and $\mathrm{F}$ describe strongly evaluated complex ones and most of the flares produced by these evaluated groups. Note that in the calculation of daily sunspot number all SGs have the same weight.

All solar activity indicators show a periodic behavior with period ranging from days to hundreds of years. The best-known solar periodic variations are the eleven year sunspot cycle and the 27-day solar rotation periodicity that is induced by large long-lived ARs with lifetimes longer than one solar rotation. Investigations of possible periodicities other than these two periods have been of interest for a long time. Let us recall these studies; in 1984, Rieger (Rieger et al., 1984) was the first to reveal a 158-day periodicity in the Sun, while studying $\gamma$-ray flare data from Solar Maximum Mission (SMM) in solar cycle 21 (C21). Approximately, the same periodicity was also discovered in X-ray flares data taken from the Geosynchronous Operational Environmental Satellites (GOES) for the same solar cycle (Rieger et al., 1984). This was only the beginning in a sequence of relevant investigations. In the context of these attempts, it is notable that apart from the Rieger periodicity itself, numerous other periodicities were discovered, such as 128, 102, 78 and 51-day periodicities (Bai and Sturrock, 1991; Bai, 1992). Consequently, Rieger periodicity was connected to highly energetic flares which are presumably triggered by the emergence of photospheric magnetic flux with the same period (Ballester, Oliver, and Baudin, 1999; Ballester, Oliver, and Carbonell, 2002). The most important short-time periodicities in different kinds of solar activity, as reported before, are as follows: i) a 153-day period in $\mathrm{H} \alpha$ flare importance (Ichimoto et al., 1985), hard X-ray peak rate (Dennis, 1985; Bai and Sturrock, 1987; Verma et al., 1991), in flare index (Ozguc and Atac, 1989, 1994; Ozguc, Atac, and Rybak, 2003), 10.7-cm radio peak flux (Kile and Cliver, 1991), and production of energetic electrons in the interplanetary space (Droege et al., 1990); ii) 323- and 540-day periodicities in daily sunspot number and sunspot area (Oliver, Carbonell, and Ballester, 1992) in flare index (Kilcik et al., 2010); iii) a quasi-period of about 160 days in the photospheric magnetic flux (Ballester, Oliver, and Carbonell, 2002); iv) $157 \pm 11$ day periodicity in X-ray flares greater than M5 (Lou et al., 2003).

On the other hand, large-scale solar activity is produced by the energy stored active region magnetic fields. Consequently flares often occur at sites in active regions overlying neutral lines where fields are strongly sheared. Observations show that the photospheric topology of the magnetic field is one of the key factors in determining the evolution of ARs. There is a general trend for large ARs to produce large flares and for more complex ARs to generate more numerous and larger flares than other ARs of similar size (Sammis, Tang, and Zirin, 2000). Here, we separated SGs in two categories based on their ARs flare productivity: if a sunspot group produced any flare (C, M, and X class flares) during its evolution we count it as a flaring AR, otherwise we count it as a non-flaring AR. Then, we analyzed temporal and periodic behavior of the sunspot counts (SSCs) in these ARs and in an attempt to more comprehensively describe general characteristics of flaring and non-flaring ARs for the last two solar cycles (1996 through 2016). We hope, this study will bring better understanding of the solar cycle, the background physical phenomena, and consequently it may increase the knowledge about Sun-Earth interactions. 


\section{Data and Analysis Method}

In this study, we investigate temporal and periodic variations of sunspot counts depending on the flare production of ARs. The group classification and the flare data are only available since August 1996. Therefore, the analyzed time interval covers only solar cycle 23 (1996 through 2008) and the ascending and maximum phases of current cycle 24 (2009 through 2016). The raw data are taken from the Space Weather Prediction Center (SWPC) 1 . These data sets include all X-Ray flares and AR informations. The daily total sunspot counts for flaring and non-flaring ARs were calculated. To compare the temporal variations of sunspot counts (SSCs) in flaring and nonflaring ARs for the investigated time period the monthly mean values were calculated. Then, to remove the short term fluctuations and reveal the long term trend a 12 steps running averaging was applied.

To reveal periodic variations in the SSC data two period analysis methods, Multitaper Method (MTM) and Morlet Wavelet Analysis, were applied with red noise approximations and 95\% confidence level. Both method have previously been successfully used analysis of solar data (Prestes et al., 2006; Kilcik et al., 2010; Mufti and Shah, 2011; Deng et al., 2013; Choudhary et al., 2014; Kilcik et al., 2014b, 2016, and reference therein).

To obtain the periodicities with high confidence level we used the MTM analysis method which enables us to detect low-amplitude harmonic oscillations in relatively short time-series with a high degree of statistical significance rejecting larger amplitude harmonics if the F-test (variance test) fails. This feature is an important advantage of the MTM over other classical methods (for details see, (Jenkins and Watts, 1969; Ghil et al., 2002)). In this study we used three sinusoidal tapers, and the frequency range was chosen from 0.0018 to 0.04 (i.e. $25-546$ days).

To localize the periodicities obtained from the MTM analysis we applied the Morlet Wavelet analysis method to the daily SSC data separately for flaring and nonflaring ARs. The Morlet wavelet is a complex sine wave, localized within a Gaussian window (Morlet et al., 1982). The method is a powerful tool for analyzing localized power variations within a time series (Torrence and Compo, 1998). The standard Interactive Data Language (IDL) packages for Morlet wavelet analysis were used, and the scalograms were obtained to study both the presence and evolution of the periodicities.

\section{Results}

Figure 1 shows the temporal variations of SSCs in flaring and non-flaring ARs for the investigated time interval (from 1996 to 2016). In general, the monthly means of the daily total SSCs in the flaring ARs are four times larger than the non-flaring ones. We emphasize that the SSCs in both data sets follows sunspot

${ }^{1} \mathrm{ftp}: / /$ ftp.swpc.noaa.gov/pub/warehouse/ 


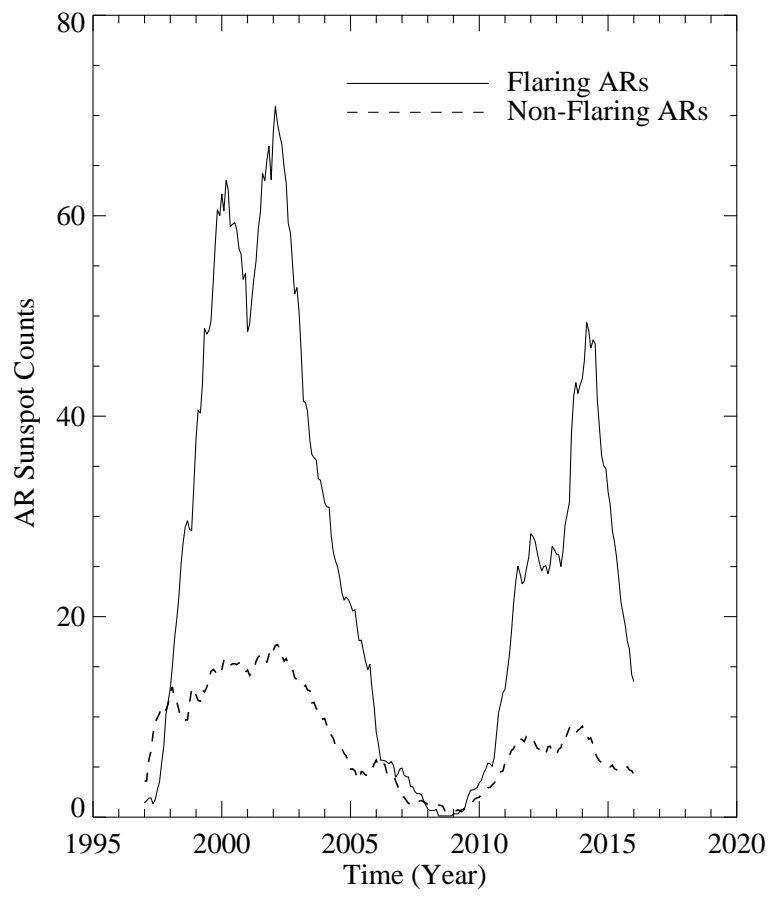

Figure 1. Temporal variations of SSCs in flaring (solid line) and non-flaring (dashed line) ARs for 1996-2016 time period.

cycle with some differences: First, SSCs in the non-flaring ARs show a wider temporal profile than that of the flaring ARs. Second, SSCs in the non-flaring ARs have multiple peaks during the maximum phase of solar cycle 23, while the flaring ARs have two prominent peaks at the same phase. Third, SSCs in the non-flaring ARs have comparable magnitude of the first and second peaks in the current solar cycle, while the first peak is almost not existent in case of the flaring ARs. Finally, there is a well defined peak in SSCs of the non-flaring ARs during 2006-2007, while that peak is not so prominent in case of the flaring ARs.

Figure 2 and 3 show observed periods of SSCs in flaring and non-flaring ARs and their localizations during the solar cycle 23. Detected periods are quite different in both MTM spectrum and wavelet scalograms: i) the largest detected period in the flaring ARs is 113 days, while there are much higher periodicities (327, 312, and 256 days) in non-flaring ARs, ii) there is a very prominent 45 day peak in non-flaring ARs, which does not appear in flaring ARs. The only similarity between the periodicities of the two data sets is the existence of solar rotation periods (27-35 days). We note that all periods detected with the MTM appear in the wavelet scalograms, but significance level of some periodicities is below the $95 \%$ confidence level.

Figure 4 and 5 show the SSCs periodic variations for the ascending and the maximum phases of solar cycle 24 which also show remarkable differences 

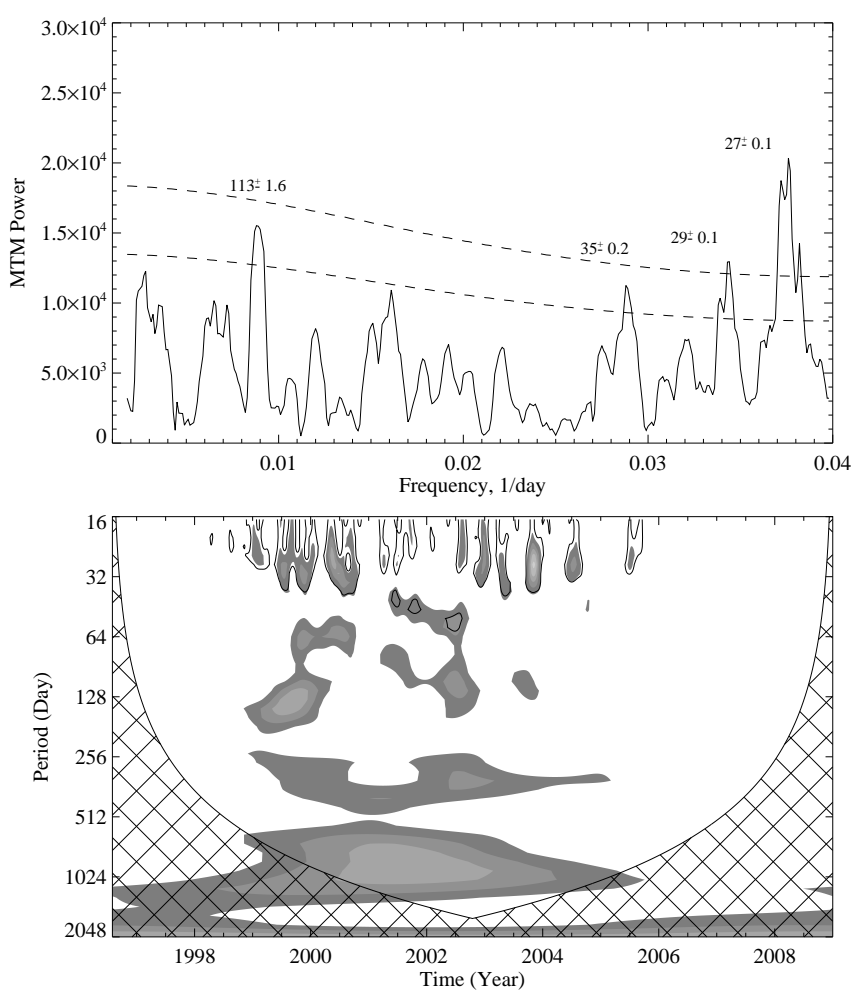

Figure 2. The multi-taper method (MTM, upper panel) and the Morlet wavelet (lower panel) analysis results for SSCs in flaring ARs during the solar Cycle 23 (1996-2008). The main peaks are labeled with the period in days in the MTM power spectrum. The horizontal dashed lines indicate the $95 \%$ and $99 \%$ confidence levels. The black contours in the wavelet scalogram indicate the $95 \%$ confidence level and the hatched area below the thin black line is the cone of influence (COI).

between the flaring and non-flaring ARs: There are no meaningful periodicities in MTM spectrum of flaring ARs exceeding 45 days, while we detect 72 day periodicity in non-flaring ARs. Note that some larger periodicities (about 250 and 600 days) appear in the wavelet scalograms of non-flaring ARs. Again solar rotation periodicities exist in both cases.

We also noted that in general, the appearance of all well defined periodicities (except the solar rotation period) are shifted toward the late phase of both cycle wavelet scalograms of the flaring ARs, while the non-flaring ARs do not show such a shift.

In Table 1, we list significant periods that appeared in flaring and non-flaring ARs in solar cycles 23 and 24 . The table shows five significant points: i) large periods (>113 day) exist only in the SSCs of non-flaring ARs, ii) a 113 day periodicity appear only in flaring ARs of solar cycle 23, iii) 72 and 55 days periods exist only in the non-flaring ARs of current cycle 24 , iv) a 45 day periodicity 

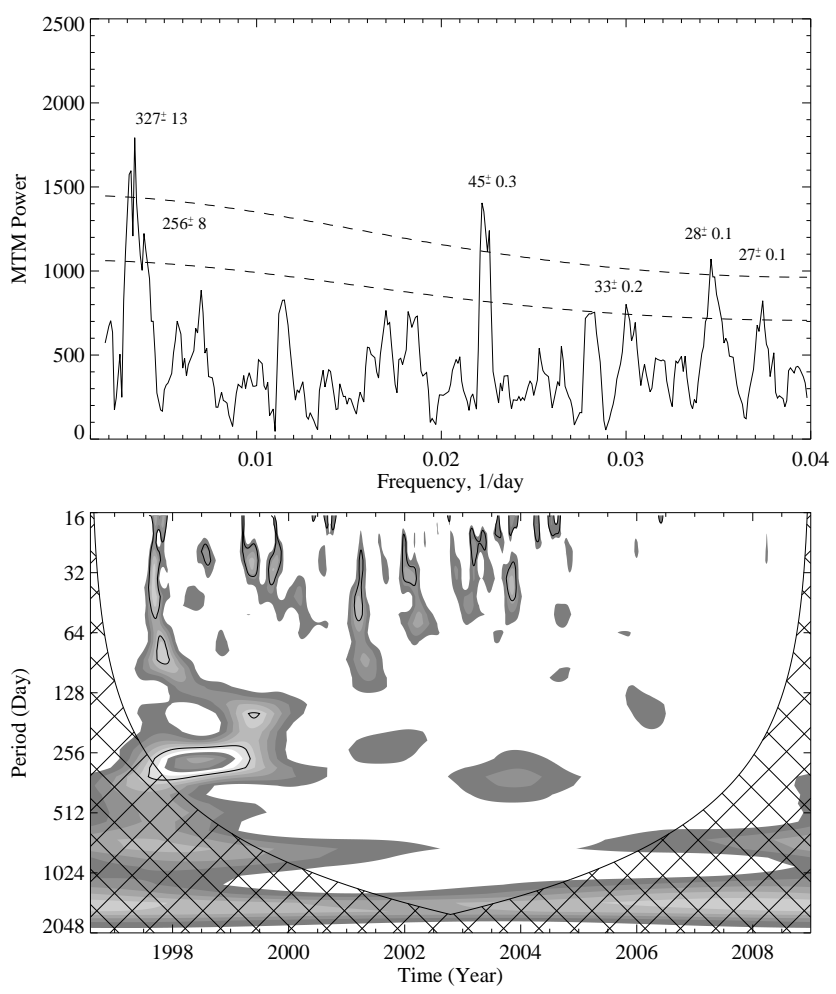

Figure 3. Same plot as in Figure 2 for non-flaring ARs.

appear only in flaring ARs of both solar cycles (Cycles 23 and 24), v) 26-35 days solar rotation periodicity exist in all cases.

\section{Conclusions and Discussions}

Here we analyzed temporal and periodic behavior of SSCs in flaring and nonflaring ARs and in an attempts to better explain different behaviors of these ARs for the last two solar cycles (1996 through 2016). We have two main findings.

i) The temporal variation of monthly means of daily total SSCs in flaring and non-flaring ARs are different and these differences are also varying from cycle to cycle; temporal profile of non-flaring ARs are wider than the flaring ones during the solar cycle 23, while they are almost the same during the current cycle 24 . The second peak of flaring ARs are strongly dominate during current cycle 24, while this difference is not such a remarkable during solar cycle 23. SSCs in the non-flaring ARs have comparable magnitude of the first and second peaks in the current solar cycle, while the first peak is almost not existent in case of the flaring ARs 

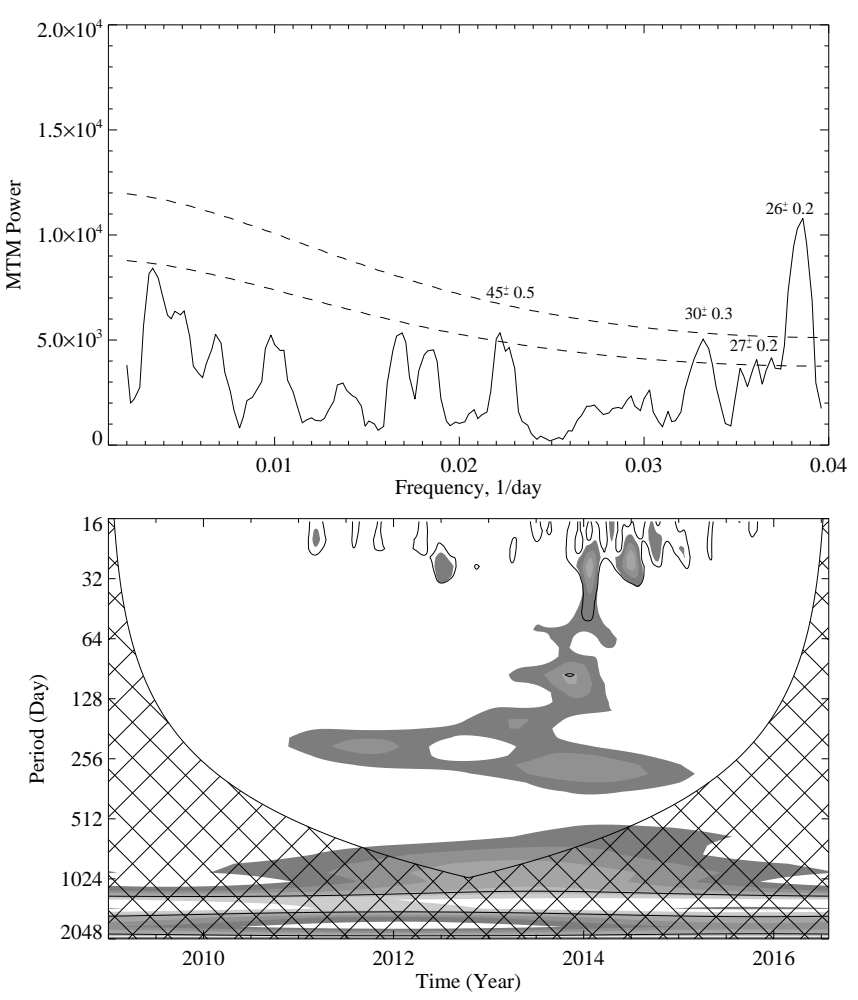

Figure 4. The multi-taper method (MTM, upper panel) and the Morlet wavelet (lower panel) analysis results for SSCs in flaring ARs during the solar Cycle 24 (2009-2016). The main peaks are labeled with the period in days in the MTM power spectrum. The horizontal dashed lines indicate the $95 \%$ and $99 \%$ confidence levels. The black contours in the wavelet scalogram indicate the $95 \%$ confidence level and the hatched area below the thin black line is the cone of influence (COI).

ii) Periodic variations observed in SSCs of flaring and non-flaring ARs are quite different in both MTM spectrum and wavelet scalograms and these variations are also different from cycle to cycle; the largest detected period in the flaring ARs is 113 days, while there are much higher periodicities $(327,312$, and 256 days) in non-flaring ARs. There are no meaningful periodicities in MTM spectrum of flaring ARs exceeding 45 days during current cycle 24, while a 113 days periodicity detected from flaring ARs of solar cycle 23. For the nonflaring ARs the largest period is 72 days during current cycle 24, while the largest period is 327 days during solar cycle 23 .

\subsection{Temporal variation of flaring and non-flaring active regions}

In the recent years, many studies were focused on the understanding of temporal variation of solar activity indicators (e.g. SSCs, SSAs, Solar X-Ray flares) in various categories and found that these variations change during a solar cycle and 

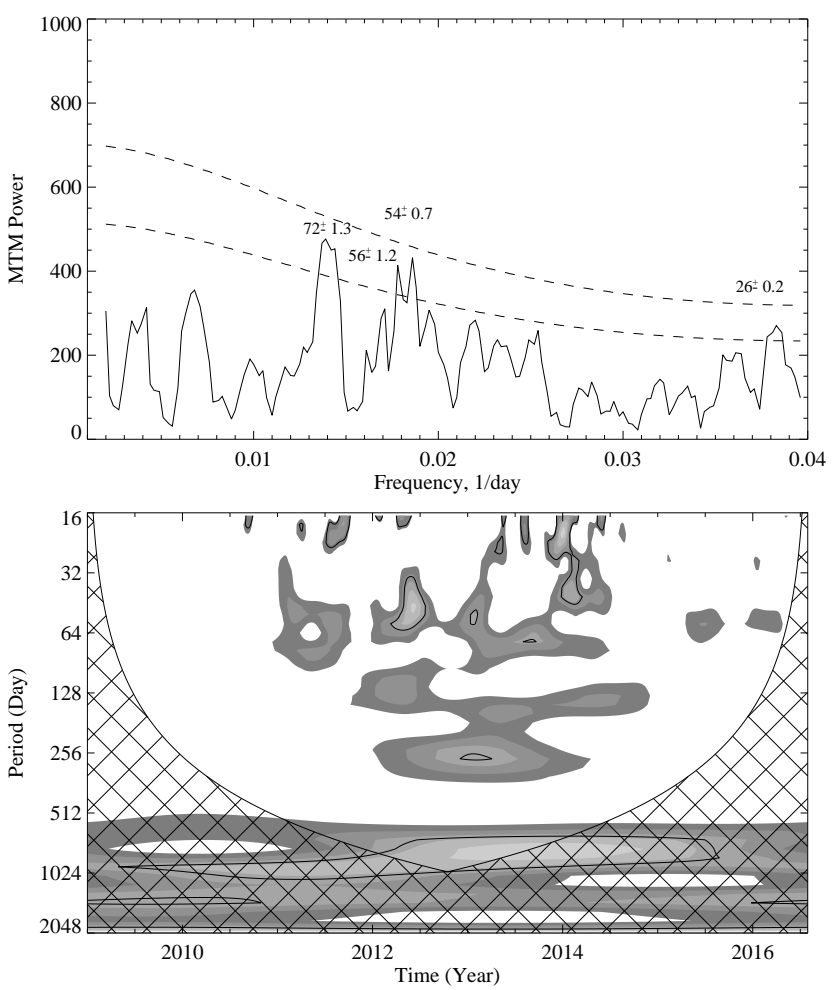

Figure 5. Same plot as in Figure 4 for non-flaring ARs.

Table 1. Periods obtained from the MTM analysis. The first column corresponds to the obtained periods and the rest of the columns show the presence of these periods in all cases by means of their significance levels.

\begin{tabular}{ccccc}
\hline \multirow{2}{*}{ Period } & \multicolumn{2}{c}{ Flaring ARs } & \multicolumn{2}{c}{ Non-flaring ARs } \\
\cline { 2 - 5 } & Cycle 23 & Cycle 24 & Cycle 23 & Cycle 24 \\
\hline $315-327$ & - & - & $+>95$ & - \\
\hline 293 & - & - & $+>95$ & - \\
\hline 256 & - & - & $+>95$ & - \\
\hline 113 & $+>95$ & - & - & - \\
\hline 72 & - & - & - & $+>95$ \\
\hline $54-56$ & - & - & - & $+>95$ \\
\hline 45 & $+>99$ & $+>95$ & - & - \\
\hline $26-63$ & $+>95$ & $+>95$ & $+>95$ & $+>95$ \\
\hline
\end{tabular}


from one cycle to another. Kilcik et al. (2011) first separated sunspot groups in two categories as small and large and analyzed the temporal variation of the SG numbers for the last four solar cycles (Cycles 20, 21, 22 and 23). They conclude that these two categories behave differently during a cycle. In the following years, these different behavior of sunspot groups are confirmed by different authors from different solar activity indicator data sets (Lefevre and Clette, 2011; Gao, Shi, and Li, 2012; Nagovitsyn, Pevtsov, and Livingston, 2012; Javaraiah, 2013; Gomez, Curto, and Gras, 2014; Kilcik et al., 2014a, b; Gao and Zhong, 2016, and reference therein). Here, we separated sunspot groups in two categories as flaring and non-flaring ARs according to their flare production. Then, we investigated the temporal variations of sunspot counts in these ARs for the last two solar cycles. We found that flaring and non-flaring ARs behave differently for different cycles; flaring ARs have remarkable peaks at the second peaks of both solar cycle 23 and 24, while these two peaks have almost the same amplitude in case of the non-flaring ARs during the both cycles. SSCs in the non-flaring ARs show a wider temporal profile than that of the flaring ARs. Also SSCs in the nonflaring ARs have multiple peaks during the maximum phase of solar cycle 23, while the flaring ARs have two prominent peaks at the same phase.These results show that the flaring ARs are better describe the large sunspot groups compared to non-flaring ones. Thus, we confirm that the most of the flare activity occur in large and complex SGs and further conclude that the temporal variations of flaring and non-flaring ARs are quite different.

\subsection{Periodic variation of flaring and non-flaring active regions}

Lean and Brueckner (1989) found a 323 day periodicity in various solar activity indicators which were the sunspot-blocking function, Zurich sunspot numbers, F10.7, and CaII K plage index. They concluded that this periodicity can have a real solar origin. Later Kilcik et al. (2014a) separated sunspot groups in four categories as small (A and B), medium (C), large (D, E, F) and final (H Modified Zurich Classes) and investigated the periodic variation of SSCs in these categories. They found this periodicity as $315-348$ days in all categories except simple ones (A and B Zurich classes). Recently, Kilcik et al. (2016) analyzed periodicities of the central latitude of SSGs (active latitude) and found about 300-370 days periodicities by using of MTM and the Morlet wavelet analysis techniques for the South hemisphere of solar cycle 18, the North hemisphere during solar cycle 20 and both North and South hemispheres of solar cycle 23. Here we analyzed SSCs periodicities in flaring and non-flaring ARs and found that this periodicity exist only in SSCs of non-flaring ARs of cycle 23.

293 and 256 days periodicities found in this study were also reported previously by different authors (Sello, 2003; Lou et al., 2003; Kilcik et al., 2010, and reference therein). Chowdhury, Khan, and Ray (2009) have reported a 261 day period from sunspot areas by means of Scargle periodogram and Morlet wavelet transform for cycles 22 and 23. Lou et al. (2003) have found these periods as $259.48 \pm 24.23$ days by applying the Fourier power spectral analysis to X-ray flares exceeding M5 class during the maximum phase of Cycle 23 (1999 - 2003), Scafetta and Willson (2013) were also found a 293 day periodicity from the TSI 
data by using a multiscale dynamical spectral analysis technique from 2003.15 to 2013.16 (descending phase of Cycle 23 and ascending phase of Cycle 24). Here we found that these two periods also exist in non-flaring active regions of solar cycle 23. Note that about 256 days period appear in the wavelet scalogram of SSCs in non-flaring ARs of current cycle 24, but its significance level is below the $95 \%$ confidence level in the MTM spectrum. Thus we may suggest that these large periodicities may arise from the non-flaring well developed SGs.

Lou et al. (2003) found $122.19 \pm 4.88$ day periodicity by means of the Fourier power spectra analysis of X-ray flares exceeding M5 class during the maximum phase of cycle 23 (1999 - 2003). Ozguc, Atac, and Rybak (2002) have investigated the periodicity of solar $\mathrm{H}$-alpha flare index during the ascending branch and the maximum phase of solar cycle 23, and reported that the 116- and 125-day periodicities are in operation during the investigated time period. Later, Kilcik et al. (2010) analyzed H-alpha solar flare index data by using MTM and Morlet wavelet analysis techniques for 1976-2007 time intervals. They investigated the cyclic variation of whole data and each cycle (Cycle 21, 22, and 23) separately and found a 113 day period from whole and cycle 23 data. Later, Kilcik et al. (2014b) found this period in the SSCs of the simple and final groups. We found that this periodicity only appears in the flaring ARs of solar cycle 23. Thus, we confirm above results and further argue that this periodicity is one of the characteristic periodicity of solar flares and also flaring ARs.

We detected 72 and 54-56 day periods from only non-flaring ARs of current cycle 24. Those periods are also reported in the literature by different authors (Zieba et al., 2001; Bai, 2003; Ozguc, Atac, and Rybak, 2003; Dimitropoulou, Moussas, and Strintzi, 2008; Kilcik et al., 2010; Chowdhury, Jain, and Awasthi, 2013; Kilcik et al., 2014b, 2016, and reference therein). Kilcik et al. (2010) analyzed the periodicity of $\mathrm{H}$-alpha flare index data for the solar cycle 21, 22, and 23. They detected these periodicities from solar cycles 21 and 22, but not from solar cycle 23 data. Scafetta and Willson (2013)analyzed the TSI data by using a multiscale dynamical spectral analysis technique from 2003.15 to 2013.16 (descending phase of Cycle 23 and ascending phase of Cycle 24) and detected a 73 day period. Recently, Kilcik et al. (2014b) analyzed the periodic variations of SSCs in different categories and found these two periodicities; a 73 day periodicity found in all categories except final ( $\mathrm{H}$ groups) ones, and a 53 day periodicity detected in all categories. Note that about 55 day period exist in all situations but their significance level just below the $95 \%$ confidence level, also it appears in the wavelet scalograms.

We detect a 45 day period from SSCs of flaring ARs of both solar cycle (Cycle 23 and 24). This periodicity also reported previously by different authors (Bai, 2003; Lou et al., 2003; Lara et al., 2008; Dimitropoulou, Moussas, and Strintzi, 2008; Kilcik et al., 2010, 2014b, 2016, and reference therein). Lou et al. (2003) investigate periodicities of X-Ray solar flares by using Fourier power spectral analyses method and Morlet wavelet analysis from 1999 to 2003 and found about 42 days period. Later, Lara et al. (2008) investigate the short term periodicity of number of coronal mass ejection by means of the maximum entropy method (MEM) and wavelet analysis during solar cycle 23 and found about 45 day period. Recently, Chowdhury et al. (2015) studied quasi-periodic variations of 
SSA/SSN, $10.7 \mathrm{~cm}$ solar radio flux, average photospheric magnetic flux by means of Lomb-Scargle periodogram and Morlet wavelet analyses during solar cycle 24 (from 2009 January to 2013 August). They detected 43 and 45 day periods from SSA and SSNs and 45 day period from $10.7 \mathrm{~cm}$ solar radio flux, but not in the average photospheric magnetic flux data. We confirm above results and further found that a 45 day period is only appearing in the flaring ARs data of both solar cycles (Cycles 23 and 24). Thus we may conclude that this period is one of the fundamental periods of only flaring ARs and it may be used as a separator between flaring and non-flaring ARs.

26-35 days solar rotation periodicity reported in the literature by many authors from different solar activity indicators (Bouwer, 1992; Bai, 2003; Kilcik et al., 2010; Chowdhury and Dwivedi, 2011; Kilcik et al., 2014b, 2016, and reference therein). This periodicity does not show any AR preference. It can be expected because the life time of many evaluated SGs are longer than one solar rotation. Therefore those SGs may appear longer than one solar rotation and affect the observed daily SSCs. Slight variations could also occur if the active region had a slow longitudinal movement ahead of, or lagging behind the rotation.

\subsection{Physical interpretation}

Parker (1955)'s dynamo equations, which can be rigorously derived from the general mean-field dynamo equations are today the most common scheme of magnetic field generation (Fioc, Nesme-Ribes, and Sokoloff, 1996; Krause and Radler, 1980). In this scheme, the dipole magnetic field is transformed by the differential rotation into near-equatorial local field of active regions ( $\Omega$ branch dynamo). At the second step the polar field is restored from the local fields (branch $\alpha$ ). This assumes one cell of meridional circulation. Field transport from the pole to the equator occurs at the lower boundary of the convection zone (tachocline), while the movement from equator to pole just below the surface.

This is a greatly simplified scheme and is currently undergoing considerable criticism and is complemented by a variety of details. In particular, it is unclear how magnetic field generated at the base of the convection zone rises up, and in which form it is made available to the observations at the photosphere level. Nevertheless, an important argument in favor of this branch of the dynamo is the high correlation between the polar field at the minimum of the cycle and the next maximum (Obridko and Shelting, 2008).

But why change the polar field? The process of restoration of the polar field to the end is not clear. The most common mechanism is of Babcock-Leighton (Babcock, 1961; Leighton, 1969; Upton and Hathaway, 2014). However still unclear, what is the ratio of two processes - advection and diffusion (Georgieva, 2011; Obridko, Nagovitsyn, and Georgieva, 2012).

Some of the difficulties in the mechanism of Babcock-Leighton are that the total magnetic flux of sunspots varies over the 11-year cycle of 10-12 times, while the large-scale flux - less than 2 times. Moreover, the total magnetic flux of sunspots equal not more than 11-14\% of the total solar magnetic flux (Harvey, 
1996). In addition, the meridional flow greatly vary over the cycle, and perhaps are not the cause but the consequence of wave activity.

The simplest scheme suppose that the generated magnetic field emerge from the depth to the surface in the form of tubes. However, modes of active regions appearance do not look like something that should be as a floating monolith tubes. We observe clearly picking up the individual cells of the magnetic field to the general structure (Getling, Ishikawa, and Buchnev, 2015, 2016). Thus one cannot directly identify the magnetic flux that is generated at great depths, and the number and size of active regions, although they are of course linked.

At present, already there is a lot of evidence to suggest that there should be, an additional process that converts the generated at greater depths large scale magnetic field in the field of sunspots. This could be distributed dynamo, subsurface dynamo, small-scale dynamo. There are reasons to believe that in the convective zone, there are not one but two or even three cells of generations (Pipin, 2014; Pipin and Kosovichev, 2014, 2015; Du, 2015; Yadav et al., 2015). Apparently, there are other processes that collect emerged weak field on the surface to strong fields of active regions (Getling, Ishikawa, and Buchnev, 2015, 2016; Brandenburg, Rogachevskii, and Kleeorin, 2016).

As a result of the presence of two related but different processes there are two important conclusions. Firstly, because of the large number of external parameters difficult to expect a complete coincidence of two cycles. Initial generation of the magnetic field provides a relatively stable 11- year variation. However, the dependence of the internal activity on the phase of the cycle can vary greatly. This also applies to the spectral composition of solar activity variations. On the one hand, you can expect the existence of a rich spectrum of periodicity, the individual frequencies can appear and disappear at different phases of the cycle. This is what is observed in results of this paper.

Second, it was to be expected that there are two populations of sunspots. This conclusion is now fully confirmed (Bludova, Obridko, and Badalyan, 2014; Obridko and Badalyan, 2014; Nagovitsyn et al., 2016). Populations of large and small groups have different statistical properties. The non-flaring active regions correspond as a rule to the small size of AR and determined by the primary dynamo mechanism. That is why the hump was observed during the declining phase of solar cycle 23 (in 2005-2006). This maximum was observed by us earlier, and its nature is studied in detail in the paper (Obridko et al. (2012), see Fig.1).

By virtue of wealth and temporal instability of the spectrum does not make sense to discuss the nature of each peak in the spectrum. It can be noted that the periods are divided into two groups. The first group periods around 1 year undoubtedly associated with large-scale fields, they seem connected with quasibiennial oscillations (QBO). It is known that QBO have a fine structure and essentially represent a set of pulses with a typical repetition rate of 1 to 3 years. This whole set periods could not be reflected in the observed spectra due to the short length of realization. They are best expressed in cycle 23, where large-scale field were more powerful.

Periods less than 100 days are clearly visible at 23 and 24 cycles in both types of groups. They appear better in the period of the cycle maximum. Separately should be said about the period of 113 days, which is shown only in the more 
powerful 23 cycle and only in flaring (that means apparently larger) groups. One can make a conservative assumption that it is related to the characteristic time of the existence of large activity complexes 4-5 solar rotations.

Therefore we can suggest that the classical dynamo generates a distributed large-scale field, while the additional dynamic process gives rise to the appearance of active regions. This second process does not end with the formation of the active region, but is expressed in a variety of relative movements of sunspots and the emergence of a magnetic flux. Thus, the flare is a natural extension of the process of formation of the active region. In the active regions where the second process has stopped, the active regions are formed less effectively and retain the statistical properties of the large-scale field.

Acknowledgments All flaring and non-flaring AR data used in this study were taken from Space Weather Prediction Center (SWPC). The wavelet software was provided by C. Torrence and G. Compo, and is available at http://paos.colorado.edu/research/wavelets/. The MTM analysis software was downloaded from http://research.atmos.ucla.edu/. This study was supported by the Scientific and Technical Council of Turkey by the Project of 115F031. V. Yurchyshyn acknowledges support from AFOSR FA9550-15-1-0322 and NSF AST-1614457 grants and KASI. JPRO acknowledges the International Space Science Institute in Bern (Switzerland) for a visitor scientist grant.

\section{References}

Babcock, H.W.: 1961, The Topology of the Sun's Magnetic Field and the 22-YEAR Cycle. Astrophys. J. 133, 572

Bai, T.: 1992, The 77 day periodicity in the flare rate of cycle 22. Astrophys. J. Lett. 388(69). Bai, T.: 2003, Periodicities in Solar Flare Occurrence: Analysis of Cycles 19-23. Astrophys. J. 591(1), 406.

Bai, T., Sturrock, P.: 1987, The 152-day periodicity of the solar flare occurrence rate. Nature 327, 601.

Bai, T., Sturrock, P.: 1991, The 154-day and related periodicities of solar activity as subharmonics of a fundamental period. Nature 350, 141.

Ballester, J.L., Oliver, R., Baudin, F.: 1999, Discovery of the Near 158 Day Periodicity in Group Sunspot Numbers during the Eighteenth Century. Astrophys. J. 522, L153.

Ballester, J.L., Oliver, R., Carbonell, M.: 2002, The Near 160 Day Periodicity in the Photospheric Magnetic Flux. Astrophys. J. 566, 505.

Bludova, N.G., Obridko, V.N., Badalyan, N.: 2014, The Relative Umbral Area in Spot Groups as an Index of Cyclic Variation of Solar Activity. Solar Phys. 289(3), 1013.

Bouwer, S.D.: 1992, Periodicities of solar irradiance and solar activity indices. II. Solar Phys. $142(2), 365$.

Brandenburg, A., Rogachevskii, I., Kleeorin, N.: 2016, Magnetic concentrations in stratified turbulence: the negative effective magnetic pressure instability. New Journal of Physics 18(12).

Choudhary, D.P., Lawrence, J.K., Norris, M., Cadavid, A.C.: 2014, Different Periodicities in the Sunspot Area and the Occurrence of Solar Flares and Coronal Mass Ejections in Solar Cycle 23 - 24. Solar Phys. 289(2), 649.

Chowdhury, P., Dwivedi, B.N.: 2011, Periodicities of Sunspot Number and Coronal Index Time Series During Solar Cycle 23. Solar Phys. 270(1), 365.

Chowdhury, P., Jain, R., Awasthi, A.K.: 2013, Periodicities in the X-Ray Emission from the Solar Corona. Astrophys. J. 778(1), 9pp.

Chowdhury, P., Khan, M., Ray, P.C.: 2009, Intermediate-term periodicities in sunspot areas during solar cycles 22 and 23. Mon. Not. Roy. Astron. Soc. 392(3), 1159. 
Chowdhury, P., Choudhary, D.P., Gosain, S., Moon, Y.J.: 2015, Short-term periodicities in interplanetary, geomagnetic and solar phenomena during solar cycle 24. Astrophys. Space Sci. 356, 7.

Deng, L.H., Qu, Z.Q., Yan, X.L., Wang, K.R.: 2013, Phase analysis of sunspot group numbers on both solar hemispheres. Res. Astron. Astrophys. 13(1), 104.

Dennis, B.R.: 1985, Solar hard X-ray bursts. Solar Phys. 100, 465.

Dimitropoulou, M., Moussas, X., Strintzi, D.: 2008, Enhanced Rieger-type periodicities detection in X-ray solar flares and statistical validation of Rossby waves existence. Mon. Not. Roy. Astron. Soc. 386(4), 2278.

Droege, W., Gibbs, K., Grunsfeld, J.M., Meyer, P., Newport, B.J., Evenson, P., Moses, D.: 1990, A 153 day periodicity in the occurrence of solar flares producing energetic interplanetary electrons. ApJS 73, 279.

Du, Z.L.: 2015, Bimodal Structure of the Solar Cycle. Astrophys. J. 803(1), 15.

Fioc, M., Nesme-Ribes, E., Sokoloff, D.: 1996, Asymptotic properties of dynamo wave. Magnetohydroynamics 31, 18.

Gao, P.X., Zhong, J.: 2016, The curious temporal behavior of the frequency of different class flares. New Astron. 43, 91. DOI

Gao, P.X., Shi, X.J., Li, Y.: 2012, Cyclical behavior of solar filaments. Astron. Nachr. 333(7), 576.

Georgieva, K.: 2011, Why the Sunspot Cycle Is Double Peaked. ISRN Astronomy and Astrophysics 2011, Article ID 437878.

Getling, A.V., Ishikawa, R., Buchnev, A.A.: 2015, Doubts about the crucial role of the risingtube mechanism in the formation of sunspot groups. Adv. Space Res. 55(3), 862.

Getling, A.V., Ishikawa, R., Buchnev, A.A.: 2016, Development of Active Regions: Flows, Magnetic-Field Patterns and Bordering Effect. Solar Phys. 291(2), 371.

Ghil, M., Allen, M.R., Dettinger, M.D., Ide, K., Kondrashov, D., Mann, M.E., Robertson, A.W., Saunders, A., Tian, Y., Varadi, F., Yiou, P.: 2002, Advanced spectral methods for climatic time series. Rev. Geophys. 40,3.1.

Gomez, A., Curto, J.J., Gras, C.: 2014, Evolution of Sunspot Characteristics in Cycle 23. Solar Phys. 289(1), 91.

Harvey, K.L.: 1996, Large Scale Patterns of Magnetic Activity and the Solar Cycle. Bulletin of the American Astronomical Society 28, 867.

Hathaway, D.H.: 2009, Solar Cycle Forecasting. Space Sci. Rev. 144, 401.

Ichimoto, K., Kubota, J., Suzuki, M., Tohmura, I., Kurokawa, H.: 1985, Periodic behaviour of solar flare activity. Nature 316, 422

Javaraiah, J.: 2013, Long-term temporal variations in the areas of sunspot groups. Adv. Space Res. 52, 963.

Jenkins, G.M., Watts, D.G.: 1969, Spectral Analysis and Its Applications holden-day, london., Holden-Day, London., ???.

Kilcik, A., Ozguc, A., Rozelot, J.P., Atac, T.: 2010, Periodicities in Solar Flare Index for Cycles 21 - 23 Revisited. Solar Phys. 264(1), 255.

Kilcik, A., Yurchyshyn, V.B., Abramenko, V., Goode, P.R., Ozguc, A., Rozelot, J.P., Cao, W.: 2011, Time Distributions of Large and Small Sunspot Groups Over Four Solar Cycles. Astrophys. J. 731, 30. DOI

Kilcik, A., Yurchyshyn, V.B., Ozguc, A., Rozelot, J.P.: 2014a, Solar Cycle 24: Curious Changes in the Relative Numbers of Sunspot Group Types. Astrophys. J. Lett. 794, L2. DOI

Kilcik, A., Yurchyshyn, V.B., Ozguc, A., Rozelot, J.P.: 2014b, Sunspot Count Periodicities in Different Zurich Sunspot Group Classes Since 1986. Solar Phys. 289(2), 4365.

Kilcik, A., Yurchyshyn, V., Clette, F., Ozguc, A., Rozelot, J.P.: 2016, Active Latitude Oscillations Observed on the Sun. Solar Phys. 291(4), 1077.

Kile, J.N., Cliver, E.V.: 1991, A search for the 154 day periodicity in the occurrence rate of solar flares using Ottawa $2.8 \mathrm{GHz}$ burst data, 1955-1990. Astrophys. J. 370, 442.

Krause, F., Radler, K.H.: 1980, Mean field magnetohydrodynamics and dynamo theory, Akademie-Verlag, ???.

Lara, A., Borgazzi, A., Mendes, O., Rosa, R.R., Domingues, M.O.: 2008, Short-Period Fluctuations in Coronal Mass Ejection Activity during Solar Cycle 23. Solar Phys. 248, 155.

Lean, J.L., Brueckner, G.E.: 1989, Intermediate-term solar periodicities - 100-500 days. Astrophys. J. 337, 568.

Lefevre, L., Clette, F.: 2011, A global small sunspot deficit at the base of the index anomalies of solar cycle 23. Astron. Astrophys. 536, 4pp. 
Leighton, R.B.: 1969, A Magneto-Kinematic Model of the Solar Cycle. Astrophys. J. $156,1$.

Lou, Y.Q., Wang, Y.M., Fan, Z., Wang, J.X. S. Wang: 2003, Periodicities in solar coronal mass ejections. Mon. Not. Roy. Astron. Soc. 345(3), 809.

McIntosh, P.S.: 1990, The classification of sunspot groups. Solar Phys. 125, 251.

Morlet, J., Arens, G., Forgeau, I., Giard, D.: 1982, Wave Propagation and Sampling Theory. Geophysics 47, 203.

Mufti, S., Shah, G.N.: 2011, Solar-geomagnetic activity influence on Earth's climate. J. Atmos. Solar-Terr. Phys. 73(13), 1607.

Nagovitsyn, Y.A., Pevtsov, A.A., Livingston, W.C.: 2012, On a Possible Explanation of the Long-term Decrease in Sunspot Field Strength. Astrophys. J. Lett. 758(1), 5pp.

Nagovitsyn, Y.A., Pevtsov, A.A., Osipova, A.A., Tlatov, A.G., Miletskii, E.V., Nagovisyna, E.Y.: 2016, Two populations of sunspots and secular variations of their characteristics. Astronomy Letters 42(10), 703.

Obridko, V.N., Badalyan, N.: 2014, Cyclic and Secular Variations Sunspot Groups with Various Scale. Astronomy Reports 58(12), 936.

Obridko, V.N., Shelting, B.D.: 2008, On Prediction of the Strength of the 11-Year Solar Cycle No. 24. Solar Phys. 248, 191.

Obridko, V.N., Nagovitsyn, Y.A., Georgieva, K.: 2012, The Unusual Sunspot Minimum: Challenge to the Solar Dynamo Theory. Astronomy and Space Science Proceedings 30, 179 .

Obridko, V.N., Ivanov, E.V., Ozguc, A., Kilcik, A., Yurchyshyn, V.B.: 2012, Coronal mass ejections and the index of effective solar multipole, Solar Physics. Solar Phys. 281, 779.

Oliver, R., Carbonell, M., Ballester, J.L.: 1992, Intermediate-term periodicities in solar activity. Solar Phys. 137, 141.

Ozguc, A., Atac, T.: 1989, Periodic behavior of solar flare index during solar cycles 20 and 21. Solar Phys. 123, 357.

Ozguc, A., Atac, T.: 1994, The 73-day periodicity of the flare index during the current solar cycle 22. Solar Phys. 150, 339.

Ozguc, A., Atac, T., Rybak, J.: 2002, Flare index variability in the ascending branch of solar cycle 23. J. Geophys. Res. $\mathbf{1 0 7}(\mathrm{A} 7)$, SSH 11.

Ozguc, A., Atac, T., Rybak, J.: 2003, Temporal variability of the flare index (1966-2001). Solar Phys. 214(2), 375.

Parker, E.N.: 1955, Hydromagnetic dynamo models. Astrophys. J. 122, 293.

Petrovay, K.: 2010, Solar Cycle Prediction. Living Rev. Solar Phys. 7(1), 6.

Pipin, V.V.: 2014, Dependence of magnetic cycle parameters on period of rotation in non-linear solar-typedynamos. Mon. Not. Roy. Astron. Soc. 451(2), 1528.

Pipin, V.V., Kosovichev, A.G.: 2014, Effects of Anisotropies in Turbulent Magnetic Diffusion in Mean-field SolarDynamoModel. Astrophys. J. 785(1), 12.

Pipin, V.V., Kosovichev, A.G.: 2015, Generation and Properties of Large-Scale Nonaxisymmetric Magnetic Fields by Solar Dynamo. IAU General Assembly, Meeting No: 29.

Prestes, A., Rigozo, N.R., Echer, E., Vieira, L.E.A.: 2006, Spectral analysis of sunspot number and geomagnetic indices (1868 2001). J. Atmos. Solar-Terr. Phys. 68(2), 182.

Rieger, E., Kanbach, G., Reppin, C., Share, G.H., Forrest, D.J., Chupp, E.L.: 1984, A 154-day periodicity in the occurrence of hard solar flares? Nature 312, 623

Sammis, I., Tang, F., Zirin, H.: 2000, The Dependence of Large Flare Occurrence on the Magnetic Structure of Sunspots. Astrophys. J. 540, 583.

Scafetta, N., Willson, R.C.: 2013, Multiscale comparative spectral analysis of satellite total solar irradiance measurements from 2003 to 2013 reveals a planetary modulation of solar activity and its nonlinear dependence on the 11 yr solar cycle. Pattern Recognition in Physics 1(1), 123.

Sello, S.: 2003, Wavelet entropy and the multi-peaked structure of solar cycle maximum. New Astron. 8(2), 105.

Torrence, C., Compo, G.P.: 1998, A Practical Guide to Wavelet Analysis. Bull. Am. Meteorol. Soc. $\mathbf{7 9}(1), 61$.

Upton, L., Hathaway, D.H.: 2014, Predicting The Sun's Polar Magnetic Fields With A Surface Flux Transport Model. Astrophys. J. 780, 5.

Verma, V.K., Joshi, G.C., Uddin, W., Paliwal, D.C.: 1991, Search for a 152-158 days periodicity in the occurrence rate of solar flares inferred from spectral data of radio bursts. Astron. Astrophys. Suppl. 90, 83. 
Yadav, R.K., Gastine, T., Christensen, U.R., Reiners, A.: 2015, Formation of starspots in self-consistent globaldynamomodels: Polar spots on cool stars. Astron. Astrophys. 573, 14.

Zieba, S., Maslowski, J., Michalec, A., Kulak, A.: 2001, Periodicities in data observed during the minimum and the rising phase of solar cycle 23; years 1996-1999. Astron. Astrophys. 377, 297. 